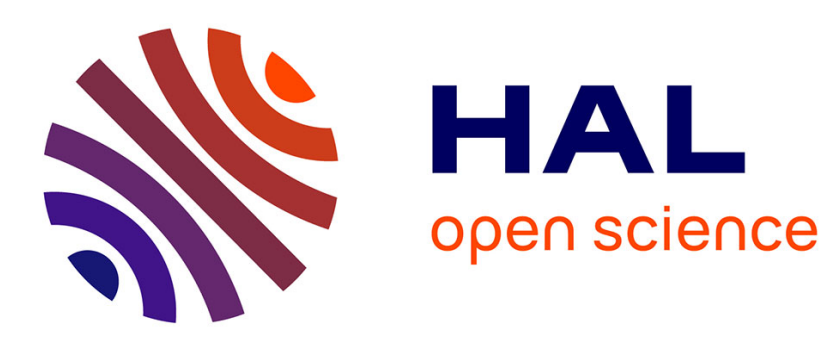

\title{
On the Improvement of Combined Fuzzy Topological and Directional Relations Information
}

\author{
Nadeem Salamat, El-Hadi Zahzah
}

\section{To cite this version:}

Nadeem Salamat, El-Hadi Zahzah. On the Improvement of Combined Fuzzy Topological and Directional Relations Information. 2010. hal-00551278

\section{HAL Id: hal-00551278 \\ https://hal.science/hal-00551278}

Preprint submitted on 3 Jan 2011

HAL is a multi-disciplinary open access archive for the deposit and dissemination of scientific research documents, whether they are published or not. The documents may come from teaching and research institutions in France or abroad, or from public or private research centers.
L'archive ouverte pluridisciplinaire HAL, est destinée au dépôt et à la diffusion de documents scientifiques de niveau recherche, publiés ou non, émanant des établissements d'enseignement et de recherche français ou étrangers, des laboratoires publics ou privés. 


\title{
On the Improvement of Combined Fuzzy Topological and Directional Relations Information
}

\author{
Nadeem SALAMAT, El-hadi ZAHZAH \\ Laboratoire de Mathématiques, Images et Applications, \\ Université de La Rochelle, France \\ $\{$ nsalam01,ezahzah $\} @$ univ-lr.fr
}

\begin{abstract}
Concept of combined extraction of topological and directional relations information developed by J. Malki et al. [1] by employing the Allen's temporal relations in $1 D$ spatial domain was improved by Matsakis and Nikitenko [2]. This latter algorithm has high computational complexity due to its limitations of object approximation and segment fuzzification.

In this paper, fuzzy Allen relations are used to define the fuzzy topological and directional relations information between different objects. Some extended results of N. Salamat and E. Zahzah [3] are discussed. Polygonal object approximation allows us to use fuzzy operators and this approach reduces computational complexity of the method for computing the combined topological and directional relations. To validate the method, some experiments are tested giving satisfactory and promising results. Affine transformation are depicted, these properties will be helpful for using the method in other areas of image analysis such as object retrieval.
\end{abstract}

Keywords: Fuzzy Allen relations, Topological and directional relations information, Computational complexity, Fuzzy operators.

\section{Introduction}

One of the fundamental tenet of modern sciences is that a phenomenon cannot be claimed to be well understood, until it is characterized in quantitative manners. Advent of digital computers have determined an expansion in the use of quantitative methods where as vagueness and imprecise knowledge information give rise to fuzzy methods. Spatial relations belong to these categories approach. Spatial relations are used for content based image retrieval (CBIR), Pattern recognition, database management, artificial intelligence (AI), cognitive science, perceptual psychology, robotics, linguistics expressions, medical imaging, image and video analysis $[4,5,6,7$, 8]. Reasoning on spatial objects needs support from representation of topological, directional, ordinal, distance relations, object size and shape.

Topological relations are derived from geometric description, while an uncertain relation is a relation which exists with a certain probability. The well-known models for finding topological relations between spatial regions are 9-intersections [9] and Region Connected Calculus (RCC) model $[10,11]$. These theories are extended to deal with fuzzy objects[12, 13, 14], in both theories, objects are modeled as fuzzy sets in $\mathbb{R}^{2}$. In 9-intersections method 44 useful topological relations were developed between simple fuzzy regions. This method also extended to 16 intersections and a set of 152 useful configurations between simple fuzzy regions in $\mathbb{R}^{2}$ is realized[15] 
while in $R C C$ number of fuzzy relations are 46 . All of these relations represent the fuzziness at object's level. Fuzziness may be present in relation's semantic while different other approaches are adopted for representing the positional information as distance and orientation.

The methods which represents such information and fuzziness in relation's semantic are discussed in $[16,17,18,19,20]$. Most of these methods work only for a particularly disjoint topological relation. The method stop working as soon as objects meet or in some cases overlap. In the methods based on force histograms [20], $2 D$ areal objects are represented by union of $1 D$ segments.

The 9-intersections model for topological relations and the $R C C$ theory have a rich support for the topological relations $[21,10]$ and provide information about a topological relation without providing information that where a topological relation exists in the space? Consider for instance that two objects overlap, both of theories provide information about the topological relation overlap, but they don't give any information about where in space the two objects overlap. As object $A$ overlaps object $B$ from north or north_west or west, or other topological relation, object $A$ disjoint from object $B$ and lies in south of object $B$. To know the relative position of object $A$ according to object $B$, we have to apply another method type, because qualitative methods provide information regarding the extended objects and they don't care about the topological relation.

Allen[22] introduced 13 interval relations in temporal domain. These relations are commonly used to represent the knowledge in artificial intelligence. Different approaches for fuzzification of Allen temporal relations are proposed such as in [23]. These temporal Allen relations are applied into the spatial domain due to homeomorphism between the temporal domain and $1 D$ spatial domain. Allen relations represent the topological relations in $\mathbb{R}$. These relations are used to answer the question that where in space a certain topological relation holds?

Allen relations are applied in a $2 D$ space by decomposition of a two-dimensional object into $1 D$ parallel segments. This decomposition process is repeated in all directions and method is applied to get combined topological and directional relations data. Combined topological and directional relations was first introduced by J. Malki et al.[1] by using the $1 D$ Allen relations. Allen relations divide the whole space into thirteen fuzzy partition and each partition corresponds to a fuzzy Allen relation. This method was further improved by Matsakis and Nikitenko[2].

This latter method is costly regarding time constraint. The aim of this paper is to reduce time consuming for the computation of combined fuzzy Allen relations information developed in[2]. Time is reduced in two ways, reducing and simplifying the number of computations and suggest an alternative way for the treatment of longitudinal section. As time constraint depends upon number of segments to be treated, to reduce the number of segments a polygonal object approximation is used. Algorithm for fuzzification of longitudinal section is replaced by fuzzy connectors. This approach decreases its time complexity from $O(n M \sqrt{M})$ to $O(n N \log (N)$, where $n$ represents number of directions, $N$ number of vertices of polygons and $M$ number of pixels to be processed

This paper is arranged as follows, next section describes some preliminary definitions, section 2 describes the terminology used, Allen relations in 1D space, fuzzification of Allen relations and finally the definition of a histogram of fuzzy Allen relations, section 3 describes the fuzzy logic connectors, treatment of a segment and longitudinal section and histogram computation with new approach, in section 4 experiments are described, time complexity for both methods are compared in section 5, in section 6 effects of different affine transformations are discussed, section 7 concludes the paper. 


\section{Preliminary definitions}

In this section we recall some basic definitions which are frequently used throughout the reminder of the paper.

Fuzzy set A fuzzy set $A$ in a set $X$ is a set of pairs $\left(X, \mu_{A}(x)\right)$ such that $A=\left\{\left(x, \mu_{A}(x) \mid x \in X\right)\right\}$

Fuzzy membership function A membership function $\mu$ in a set $X$ is a function $\mu: X \rightarrow[0,1]$. Different fuzzy membership functions are proposed according to the requirements of the applications. For instance, Trapezoidal membership function is defined as

$$
\mu(x ; \alpha, \beta, \gamma, \delta)=\max \left(\min \left(\frac{x-\alpha}{\beta-\alpha}, 1, \frac{\delta-x}{\delta-\gamma}\right), 0\right)
$$

it is written as $\mu_{(\alpha, \beta, \gamma, \delta)}(x)$ where $x, \alpha, \beta, \gamma, \delta \in \mathbb{R} \wedge \alpha<\beta \leq \gamma<\delta$.

Force histogram The force histogram attaches a weight to the $\operatorname{argument}$ object $A$ that this lies after $B$ in direction $\theta$, it is defined as

$$
\mathbf{F}^{A B}(\theta)=\int_{-\infty}^{+\infty} F\left(\theta, A_{\theta}(v), B_{\theta}(v)\right) d v
$$

The definition of Force histogram $\mathbf{F}^{A B}(\theta)$, directly depends upon the definition of real valued functions $\phi, f$ and $F$ used for the treatment of points, segments and longitudinal sections respectively[20]. These functions are defined as

$$
\left.\begin{array}{rl}
\phi_{r}(y) & = \begin{cases}\frac{1}{y^{r}} & \text { if } y>0 \\
0 & \text { otherwise }\end{cases} \\
f\left(x_{I}, y_{I J}^{\theta}, z_{J}\right) & \left.=\int_{x_{I}+y_{I J}^{\theta}}^{x_{I}+y_{I J}^{\theta}+z_{J}} \int_{0}^{z_{J}} \phi(u-w) d w\right) d u \\
F\left(\theta, A_{\theta}(v), B_{\theta}(v)\right) & =\sum_{i=1 . . n, j=1 . . m} f\left(x_{I i}, y_{I i J j}^{\theta}, z_{J j}\right)
\end{array}\right\}
$$

Where $n, m$ represents the number of segments of object $A$ and object $B$ respectively and variables $(\mathrm{x}, \mathrm{y}, \mathrm{z})$ are explained in figure 1 .

\section{Histograms of fuzzy Allen relations}

\subsection{Oriented lines, segments and longitudinal sections}

Let $A$ and $B$ be two objects. $(v, \theta) \in \mathbb{R}$, where $v$ is any real number and $\theta \in[-\pi, \pi] . \Delta_{\theta}(v)$ is an oriented line at orientation angle $\theta . A \cap \Delta_{\theta}(v)$ is the intersection of object $A$ and oriented line $\Delta_{\theta}(v)$. It is denoted by $A_{\theta}(v)$, called segment of object $A$. If there exist more than one segment then it is called longitudinal section as in case of figure 1 where $A \cap \Delta_{\theta}(v)$ has two segments, length of its projection points on x-axis is $x$. Similarly for object $B$ where $B \cap \Delta_{\theta}(v)=B_{\theta}(v)$ is segment and $z$ is length of its projection points. $y$ is the difference between the minimum of projection points of $A \cap \Delta_{\theta}(v)$ and maximum of projection points of $B \cap \Delta_{\theta}(v)$. 


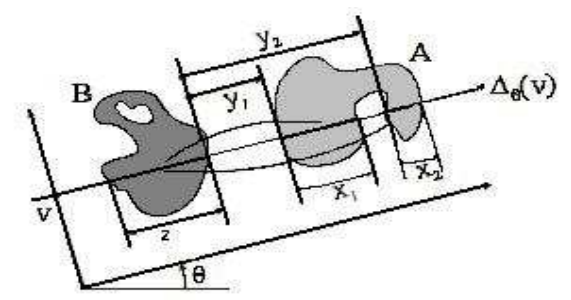

Figure 1: Oriented line, segment and longitudinal section[2]

\subsection{Allen relations in space}

Allen[22] introduced 13 mutually exclusive and exhaustive interval relations. These relations are arranged as $\mathcal{A}=\left\{<, m, o, s, f, d, e q, d_{i}, f_{i}, s_{i}, o_{i}, m_{i},>\right\}$, where $\{<, m, o, s, f, d$,$\} resp$ $\left(\left\{d_{i}, f_{i}, s_{i}, o_{i}, m_{i},>\right\}\right)$ are the relations before, meet, overlap, start, finish, during (resp the inverse relations of the cited ones). The relation $e q$ is the equality spatial relation. All the Allen relations in space are conceptually illustrated in figure 2 . These relations have a rich support for the topological and directional relations.

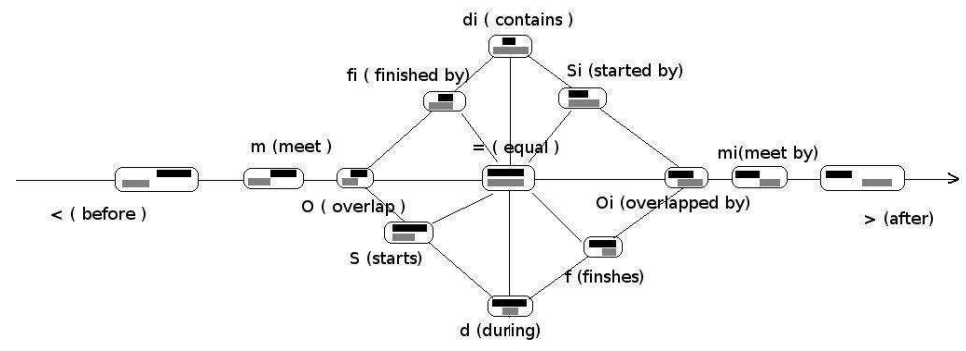

Figure 2: Black segments represent the reference objects and grey segments represent the argument objects

\subsection{Fuzzification of Allen relations}

Different approaches are used for fuzzification of Allen temporal relations, some of them use the fuzzification based on the human defined variables and fuzzification is described only for use in temporal domain, for the qualitative aspects of temporal knowledge and qualitative temporal reasoning processes. There is a homeomorphism between the Allen's temporal relations and $1 D$ topological relations in spatial domain. Due to this homeomorphism, Allen relations are also used for extracting the combined topological and directional relations information[1,2]. Fuzzy Allen relations are used to represent the fuzzy topological relations where vagueness or fuzziness is represented at the relation's level. Fuzzification of Allen relations doesn't depend upon particular choice of fuzzy membership function. Trapezoidal membership function (equation(1)) is used due to flexibility in shape. Let $r(I, J)$ be an Allen relation between segments $I$ and $J$ where $I \in A$ (argument object) $J \in B$ (reference object), $r^{\prime}$ is the distance between $r(I, J)$ and it's conceptional neighborhood. We consider a fuzzy membership function $\mu: r^{\prime} \longrightarrow\left[\begin{array}{ll}0 & 1\end{array}\right]$. The 
fuzzy Allen relations defined by Matsakis and Nikitenko[2] are

$$
\left.\begin{array}{rl}
f_{<}(I, J) & =\mu_{(-\infty,-\infty,-b-3 a / 2,-b-a)}(y) \\
f_{>}(I, J) & =\mu_{(0, a / 2, \infty, \infty)}(y) \\
f_{m}(I, J) & =\mu_{(-b-3 a / 2,-b-a,-b-a,-b-a / 2)}(y) \\
f_{m i}(I, J) & =\mu_{(-a / 2,0,0, a / 2)}(y) \\
f_{O}(I, J) & =\mu_{(-b-a,-b-a / 2,-b-a / 2,-b)}(y) \\
f_{O i}(I, J) & =\mu_{(-a,-a / 2,-a / 2,0)}(y) \\
f_{f}(I, J) & =\min \left(\mu_{(-(b+a) / 2,-a,-a,+\infty)}(y), \mu_{(-3 a / 2,-a,-a,-a / 2)}(y), \mu_{(-\infty,-\infty, z / 2, z)}(x)\right) \\
f_{f i}(I, J) & =\min \left(\mu_{-b-a / 2,-b,-b,-b+a / 2}(y), \mu_{(-\infty,-\infty,-b,-(b+a) / 2)}(y), \mu_{(z, 2 z,+\infty,+\infty)}(x)\right) \\
f_{s}(I, J) & =\min \left(\mu_{-b-a / 2,-b,-b,-b+a / 2}(y), \mu_{(-\infty,-\infty,-b,-(b+a) / 2)}(y), \mu_{(-\infty,-\infty, z / 2, z)}(x)\right) \\
f_{s i}(I, J) & =\min \left(\mu_{(-(b+a) / 2,-a,-a,+\infty)}(y), \mu_{(-3 a / 2,-a,-a,-a / 2)}(y), \mu_{(z, 2 z,+\infty,+\infty)}(x)\right) \\
f_{d}(I, J) & =\min \left(\mu_{(-b,-b+a / 2,-3 a / 2,-a)}(y), \mu_{(-\infty,-\infty, z / 2, z)}(x)\right) \\
f_{d i}(I, J) & =\min \left(\mu_{(-b,-b+a / 2,-3 a / 2,-a)}(y), \mu_{(z, 2 z,+\infty,+\infty)}(x)\right)
\end{array}\right\}
$$

where $a=\min (x, z), b=\max (x, z), x$ is the length of segment $(I)$ of argument object $A, z$ is the length of $\operatorname{segment}(J)$ of reference object $B$ and $(\mathrm{x}, \mathrm{y}, \mathrm{z})$ are computed as given in section2.1. Most of relations are defined by a single membership function and some of them by minimum of multiple membership functions like $d$ (during), $d_{i}$ (during_by), $f$ (finish), $f_{i}$ (finished_by). Two relations are directly neighbors if the fuzzy Allen relations are shared between them. If $0<r_{1}(I, J)<1$ then $0<r_{2}(I, J)<1$ such that $r_{1}(I, J)+r_{2}(I, J)=1$ and $r_{1}(I, J), r_{2}(I, J)$ are neighbors expressed in the neighborhood graph (figure2). This shows that sum of all the Allen relations is always one, from this equal $\left(f_{=}(I, J)\right.$ relation is defined.Histogram of fuzzy Allen relations represent the total area of subregions of $A$ and $B$ that are facing each other in a given direction $\theta$ [2]. Formally, this definition is

$$
\begin{gathered}
F_{r}^{A B}(\theta)=\int_{-\infty}^{+\infty} F_{r}\left(\theta, A_{\theta}(v), B_{\theta}(v)\right) d v \\
F_{r}\left(\theta, A_{\theta}(v), B_{\theta}(v)\right)=\frac{x+z}{w} \sum_{k=1}^{c} \sum_{i=1}^{m_{k}} \sum_{j=1}^{n_{k}}\left[x_{i}^{k} z_{j}^{k}\left(a_{k}-a_{k-1}\right)\right] r\left(I_{i}^{k}, j_{j}^{k}\right)
\end{gathered}
$$

Where $F_{r}^{A B}(\theta)$ represents the histogram of a fuzzy Allen relation in direction $\theta \operatorname{and} F_{r}\left(\theta, A_{\theta}(v), B_{\theta}(v)\right)$ is the representation of histogram for a given $v$ where $w=\sum_{k=1}^{c} \sum_{i=1}^{m_{k}} \sum_{j=1}^{n_{k}}\left[x_{i}^{k} z_{j}^{k}\left(a_{k}-a_{k-1}\right)\right]$, $x=\sum_{i=1}^{m_{k}} x_{i}^{k}$ and $z=\sum_{j=1}^{n_{k}} z_{j}^{k}$.

In this case $m_{k}$ and $n_{k}$ represent the total number of segments of argument object $A$ and reference object $B$ and $c$ represents number algorithm loop for fuzzification of a longitudinal section (algorithm is discussed in section 6).

\subsection{Histogram of fuzzy Allen relations}

Fuzzy Allen relation for each segment is a fuzzy set and fuzzy aggregation operators are used to combine different values of fuzzy grades. In polygonal object approximation, fuzzy Allen relations are calculated for a limited number of segments and within this region spatial relations do not change so simply generalize the given relations. This technique minimizes the 
number of segments to be treated, decreasing the temporal complexity of algorithm. This changes mathematical equation (equation (6)) histogram of fuzzy Allen relations. Formally,

$$
F_{r}\left(\theta, A_{\theta}(v), B_{\theta}(v)\right)=r\left(I_{k}, J_{k}\right)
$$

In discrete space, the integral can be written as a finite sum, thus above equation (5) for discrete space can be rewritten as

$$
F_{r}^{A B}(\theta)=(X+Z) \sum_{k=1}^{n} r\left(I_{k}, J_{k}\right)
$$

Where $Z$ is the area of reference object and $X$ is area of argument object in direction $\theta, n$ is total number of segments treated and $r\left(I_{k}, J_{k}\right)$ is an Allen relation for segment pair $\left(I_{k}, J_{k}\right)$ and $F_{r}\left(\theta, A_{\theta}(v), B_{\theta}(v)\right)$ represents a histogram of a fuzzy Allen relation.

\subsubsection{Treatment of longitudinal section}

During the decomposition process of an object into segments, there can be multiple segments for a line $\Delta_{\theta}(v)$ depending on object shape and boundary which is called longitudinal section. Different segments of a longitudinal section are at a certain distance and these distances might affect final results. In this paper, we adopt the same method as in case of force histograms. Here, we replace the sum operator in equation (3) by a fuzzy operator and use the logic connectors. This means

$$
F_{r}\left(\theta, A_{\theta}(v), B_{\theta}(v)\right)=\odot\left(f_{r}\left(x_{1}, y_{1}^{\theta}, z\right), f_{r}\left(x_{2}, y_{2}^{\theta}, z\right), \ldots ., f_{r}\left(x_{n}, y_{n}^{\theta}, z\right)\right)
$$

Where $\odot$ is a fuzzy operator and $r \in \mathcal{A}, \mathcal{A}$ is denoted in section 2.2.

\section{Example}

We introduce an example to explain different steps of the method of combining topological and directional relations information and better explains difference between method developed by Matsakis and Nikitenko[2] and the method we proposed in this paper. Let us consider two simple objects for the computation of histogram of fuzzy Allen relations. Let these simple polygonal objects be $A$ and $B$ where $A=\{(5,5),(15,5),(20,10),(20,15),(10,15)\}$ and $B=\{(5,15),(15,12),(20,20),(15,25),(10,25),(5,20)\}$ are the vertices of polygons. Computing

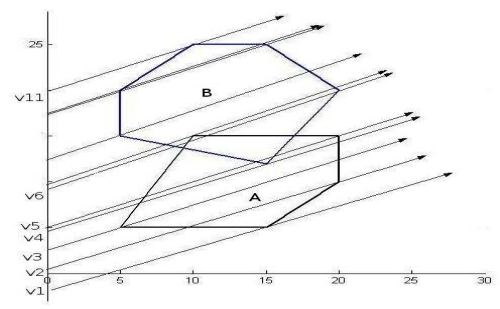

Figure 3: Showing the computation of relations by an example where each line passes through the vertex of a polygon and inclination angle is fixed at 30 degree

the histograms of Allen relation for objects $A$ and $B$ by our method involves the following steps.

1. Compute the boundary of both objects, for object $A$, line segments joining the vertices of polygon $\mathrm{A}$, in a similar way for object $B$. 
2. Fix the angle, let it be 30 degree and draw lines passing through the vertices of polygons, in our case, total number of vertices are 11 there will be 11 lines, each line passes through a vertex of a polygon.

3. Compute the intersection of line with boundary of an object, each line has one or two intersecting points with one object due to simple concave objects. In case of convex or objects with holes there may be more then two intersecting points. Consider only those lines which intersect both objects, for all the other lines relations are zero. As in figure only $\Delta_{30}(v 4), \Delta_{30}(v 5), \Delta_{30}(v 6), \Delta_{30}(v 7)$ lines intersect both objects, for other lines fuzzy Allen relations are zero. These intersecting points are

$\Delta_{30}(v 4) \cap A=\{(6,6),(20,14)\}, \Delta_{30}(v 5) \cap A=\{(6,7),(20,15)\}$,

$\Delta_{30}(v 6) \cap A=\{(9,13),(12,15)\}, \Delta_{30}(v 7) \cap A=\{(10,15)\}$,

$\Delta_{30}(v 4) \cap B=\{(15,12)\}, \Delta_{30}(v 5) \cap B=\{(15,12),(16,13)\}$,

$\Delta_{30}(v 6) \cap B=\{(9,13),(20,20)\}, \Delta_{30}(v 7) \cap B=\{(8,14),(19,20)\}$

4. Take the projection $(\mathrm{P})$ of these points on $\mathrm{x}$-axis and calculate the values of $(x, y, z)$. Here, for line $\Delta_{30}(v 4)$ these values are $P\left(\Delta_{30}(v 4) \cap A\right)=\{6,20\}$ and $P\left(\Delta_{30}(v 4) \cap B\right)=\{15\}$, from this we get $(x, y, z)=(14,-9,0)$.

5. Compute the fuzzy Allen relation for each segment by using equation (4). This system of equations will provide us the following results for the aforementioned $(\mathrm{x}, \mathrm{y}, \mathrm{z})$ ( segments obtained by taking intersection of objects with line $\left.\Delta_{30}(v 4)\right)$.

$f_{>}\left(I_{4}, J_{4}\right)=0, f_{<}\left(I_{4}, J_{4}\right)=0, f_{m}\left(I_{4}, J_{4}\right)=0, f_{m i}\left(I_{4}, J_{4}\right)=0$,

$f_{O}\left(I_{4}, J_{4}\right)=0 f_{o i}\left(I_{4}, J_{4}\right)=0, f_{f}\left(I_{4}, J_{4}\right)=0 f_{f i}\left(I_{4}, J_{4}\right)=0$,

$f_{s}\left(I_{4}, J_{4}\right)=0 f_{s i}\left(I_{4}, J_{4}\right)=0, f_{d}\left(I_{4}, J_{4}\right)=0, f_{d i}\left(I_{4}, J_{4}\right)=1$, and $f_{=}\left(I_{4}, J_{4}\right)=0$,

6. Repeat this process for lines $\Delta_{30}(v 5), \Delta_{30}(v 6)$ and $\Delta_{30}(v 7)$ ( for other lines relations are already zero), sum all the relations and multiply the resultant relation by the sum of surface areas of two objects between line $\Delta_{30}(v 4)$ and line $\Delta_{30}(v 7)$. This will provide the number of pixels of both objects under a specific fuzzy Allen relation and for normalization, divide each fuzzy Allen by the sum of all the Allen relations for $\theta=30$ degree, this will give us the percentage area of two objects under a fuzzy Allen relation.

7. Increase the angle by one degree and repeat the above steps(2-6).

We calculate the values $(x, y, z)$ for Matsakis and Nikitenko [2]. In this method objects are considered as regular closed sets. The difference between methods is the way to compute the triplet $(\mathrm{x}, \mathrm{y}, \mathrm{z})$ and object approximation.

1. Fix the angle $\theta$ and draw the pencil of oriented lines $\Delta_{\theta}(v)$, in this case number of lines will be much greater than 11 due to pencil of lines.

2. Compute the intersection of a oriented line with both objects $A$ and $B$. Let us consider the same line $\Delta_{30}(v 4)$. Then

$\Delta_{30}(v 4) \cap A=\{(6,6),(7,7),(8,7),(9,8),(10,8),(10,9),(11,9)$,

$(12,10),(13,10),(14,11),(15,11),(15,12),(16,12),(16,13)$,

$(17,13),(18,13),(18,14),(19,14),(20,14)\}$.

Similarly for object $B, \Delta_{30}(v 4) \cap B=\{(15,12)\}$. Take projections on x-axis and compute the values of $(x, y, z)$, here the values will be the same as in above case and produce the same results. i.e., $x=14, y=-9, z=0, x$ and $z$ are the diameter of point sets on real line and $y$ is the difference between the minimum of argument point set and maximum of reference point set. Remaining process of computation is identical to our method. 
3. Compute Allen relations for every line that intersect the objects. Pencil of lines is created then same calculations are made for every line.

This example elaborates the difference between two methods, and how the new proposed method reduces the time for computation, by simplifying and limiting the number of intersections in one line and an object. Secondly, it decreases the number of lines to be treated for one direction.

\section{Fuzzy logic connectors}

In decision theory, there arise some situations where solution depends upon combination of different information provided by different sources. In such a situation, fuzzy connectors are used. In this section, different fuzzy aggregation operators (fuzzy logic connectors) are studied. Aggregation refers of combining values into the one aggregated value so that the final solution seems to be well addressed in a given fashion [24, 25]. It is a mapping $\tau:[0,1]^{n} \rightarrow[0,1]$, which combines different fuzzy grades. In literature of fuzzy set theory there exist a variety of operators such as fuzzy T-norms, $T$-conorms and so on,some commonly used operators are:

- Fuzzy OR: $\mu_{O R}(x)=\max \left(\mu_{A}(x), \mu_{B}(x)\right)$;

- Fuzzy AND: $\mu_{A N D}(x)=\min \left(\mu_{A}(x), \mu_{B}(x)\right)$;

- Fuzzy Algebraic Product: $\mu_{P R O D}(x)=\Pi_{i=1}^{2}\left(\mu_{(i)}(x)\right)$;

- Fuzzy Algebraic Sum: $\mu_{S U M}(x)=1-\Pi_{i=1}^{2}\left(1-\mu_{i}(x)\right)$;

- Fuzzy $\gamma$ operator: $\mu_{\gamma}(x)=\left[\mu_{S U M}(x)\right]^{\gamma} *\left[\left(\mu_{P R O D}(x)\right]^{1-\gamma}\right.$ where $\gamma \in[0,1]$

The $O R$ operator is actually the union or max operator, while the AND is intersection or min operator. The contribution for resultant of $O R(A N D)$ fuzzy operator is a single input value, which is maximum (minimum). For other operators, both values contribute. The fuzzy algebraic sum (product) operator makes the set result larger than or equal to maximum (resp less than or equal to minimum ) the contributing values while Fuzzy $\gamma$ operator changes the result value from minimum to maximum values depending on the choice of $\gamma$.

\section{Affine properties}

Affine properties are important in the pattern recognition especially object matching in a scene analysis. These properties of histograms of fuzzy Allen relations are depicted below which are independent from fuzzy membership functions.

- Object commutativity: Pair (A, B) be assessable $r$ is any fuzzy Allen relations and for all relations, except the relation during and during_by. $F_{r}^{A, B}(\Theta)=F_{r}^{B, A}(\Theta+\pi)$. And for relation during and during_by we have $F_{d}^{A, B}(\Theta)=F_{d i}^{B, A}(\Theta)$

- Orthogonal symmetry: Let orthogonal symmetry denoted by sym about the oriented line with slop $\alpha$ then histogram of fuzzy Allen relations: $F_{r}^{s y m(A), \text { sym }(B)}(\Theta)=F_{r}^{A, B}(2 \alpha-\Theta)$

- Central dilation: Let central dilation(scale) denoted by $d i l$ and $\lambda$ is dilation ratio then $F_{r}^{\operatorname{dil}(A), \operatorname{dil}(B)}(\Theta)=\lambda^{2} F_{r}^{A, B}(\Theta)$ 
- Stretch: Let stretch (stre) is orthogonal to $\mathrm{x}$ axis and $k$ is stretch ratio, then histogram of fuzzy Allen relations satisfies. $F_{r}^{\text {stre }(A), \operatorname{stre}(B)}(\Theta)=k F_{r}^{A, B}(\Theta)$

- Translation: Let translation denoted by trans and (trans(A), $\operatorname{trans}(\mathrm{B}))$ is assessable then following relation holds for histogram of fuzzy Allen relations. $F_{r}^{\operatorname{trans}(A), \operatorname{trans}(B)}(\Theta)=$ $F_{r}^{A, B}(\Theta)$

- Rotation: Let rotation ( rot) is a $\rho$-angle rotation and $(\operatorname{rot}(A), \operatorname{rot}(B))$ is assessable hence $F_{r}^{r o t}(A), \operatorname{rot}(B)(\Theta)=F_{r}^{A, B}(\Theta-\rho)$

\section{Comparison with Matsakis and Nikitenko method and interpretation}

In this section first we give the interpretation of results representation and then compare our approach with Matsakis and Nikitenko method [2]. For the experiment purpose 360 directions are considered. Instead of drawing pencil of lines in a direction, only those lines are considered which passes through vertices of polygons. Fuzzy Allen relations are computed for each segment, if there exit longitudinal section, then fuzzy aggregation operator is applied to obtain the resultant fuzzy Allen relation of whole object.

Each relation is associated with the grey scale value like before with black and white represents after, each relation has a different boundary color for better visualization of relations while opposite relations have the same boundary color. Opposite relations ( $m($ meet) and mi(meet_by) relations have the yellow boundary color) have the same boundary color. Interpretation of the grey level association to a relation and its boundary color is given in figure 4(a). Object $A$ has the light grey color and object $B$ is represented by dark grey. The thirteen histograms representing directional and topological relations are represented by layers and each vertical layer represents the total area of objects in that direction.

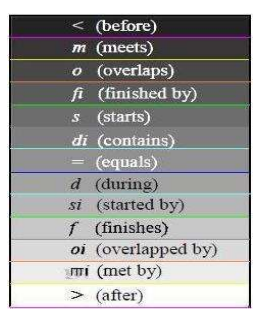

(a) Histograms representation

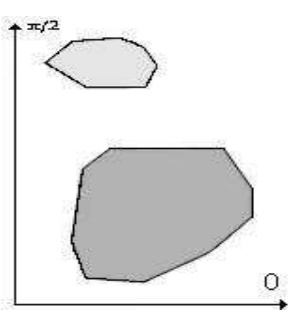

(b) Object pair

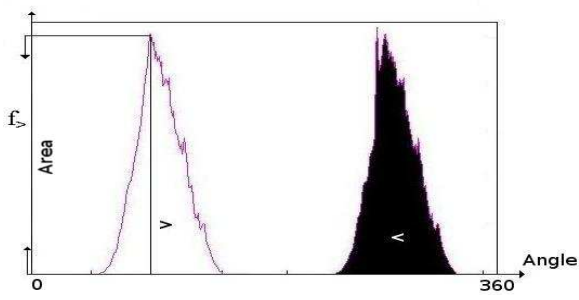

(c) Histograms

Figure 4: 4(a), Histogram representation for fuzzy Allen relations, 4(b), object pair representation and 4(c) represents histogram of fuzzy Allen relations for object pair in 4(b).

These histograms can be normalized as $A_{j}(\theta)=\frac{A_{j}(\theta)}{\sum_{i=1}^{13} A_{i}(\theta)}$ where $A_{j} \forall j=1, \ldots, 13$ is an Allen relation. This technique of normalization provide us the percentage area of both objects under a specific fuzzy Allen relation. In this example (figures in table 1) first row represents the object pair and its histograms which are not normalized, computed by our method and method developed by Matsakis and Nikitenko method (for Matsakis and Nikitenko method we copied the figure directly from [2]). The second row represents normalized histogram of fuzzy Allen relations with the respective method. Both the histograms seems similar, a small difference in the 


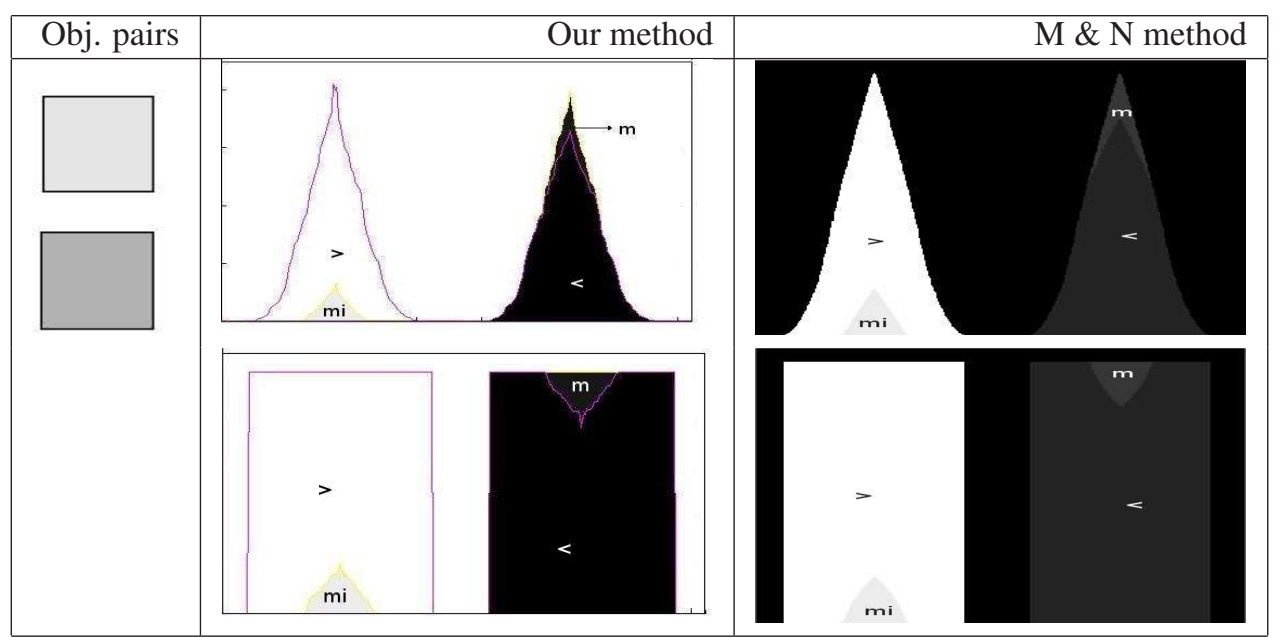

Table 1: Comparison of normalized and un-normalized histograms of fuzzy Allen relations with the Matsakis and Nikitenko and our method(axis are same as in figure 4(b) and 4(c)).

shape of a histogram represents the small change in the total surface area of two objects under the specific fuzzy Allen relations, which is less important.

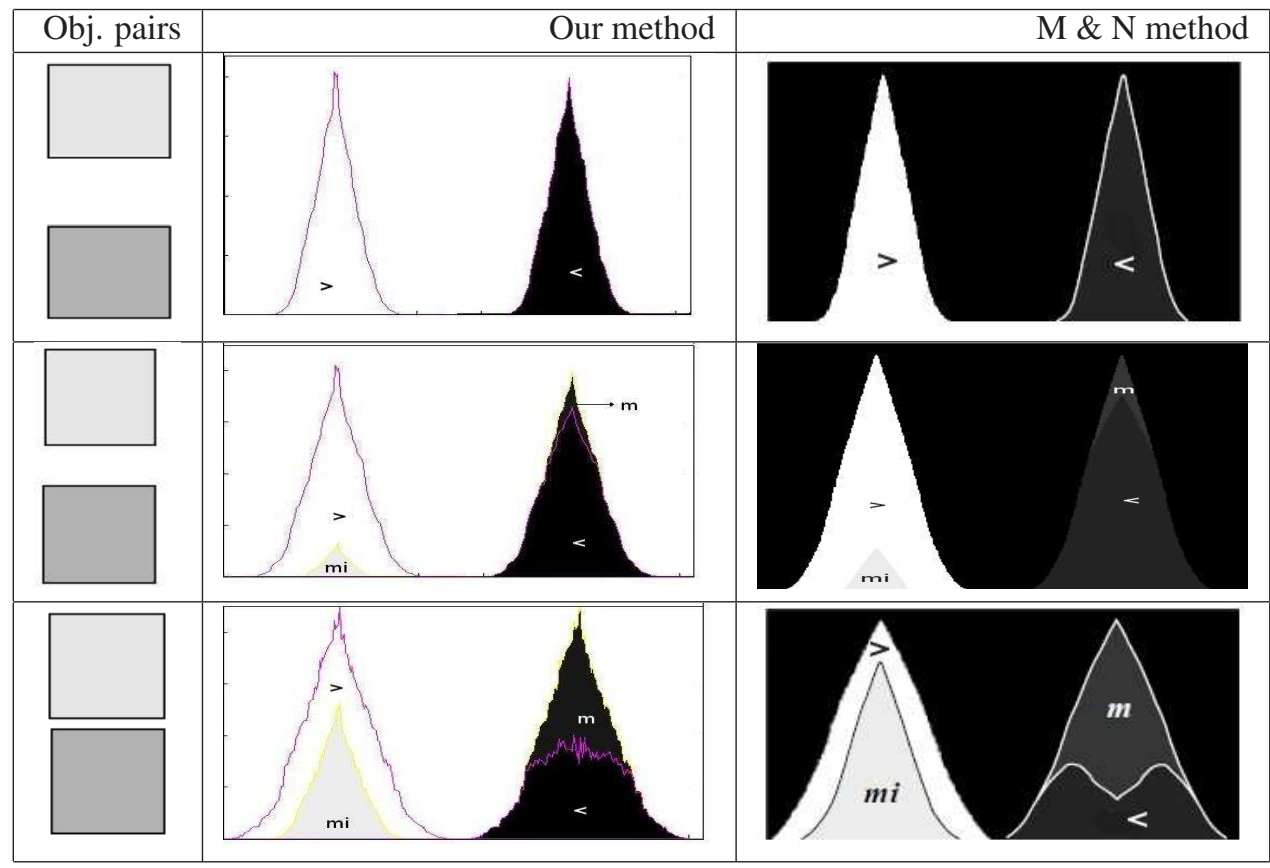

Table 2: Comparison of histograms for rectangular objects (axis are same as in figure4(b) and 4(c)). 
In this set of examples (figures in table 2), fuzzy Allen histograms are compared with the computation method of Matsakis and Nikitenko [2]. Where the first column represents object pairs at different distances, second column represents computation of histogram of fuzzy Allen relation by our method and in third column histograms are represented computed by Matsakis and Nikitenko method. These histograms of fuzzy Allen relations are distances dependant, As the objects become closer, the new histogram of fuzzy Allen relations emerge.

In both methods, similar histograms exist, there is a small difference in histogram shapes. Histogram shape represents the total area under the specific relation, as we consider object by the polygonal object approximation, this result's the small changes in object area. That is less important, more important objective is existence of a spatial relation.

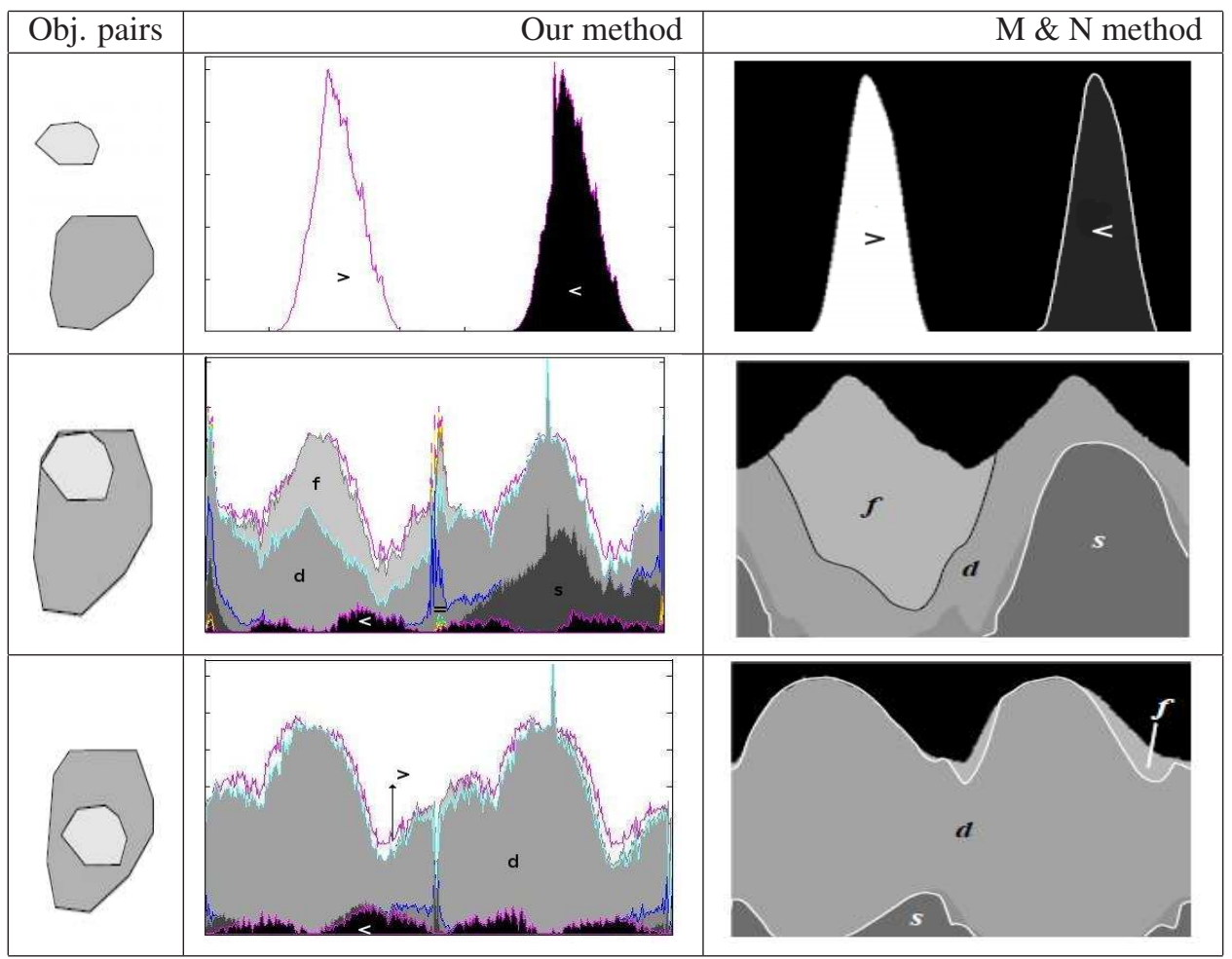

Table 3: Comparison of histograms of fuzzy Allen relations with the Matsakis and Nikitenko method and our method for the polygonal objects (axis are same as in figure 4(b) and 4(c)).

In this example (figures are shown in table 3), polygonal objects are considered. First objects are at certain distances, only after and before relation exist. Histogram resembles with the histogram computed by the Matsakis and Nikitenko method. Sharp and sudden changes in the histogram shape computed by our method are due to the problems of taking intersections in the $2 D$ digital space. Empty intersection sometime results change in the object area (sharp ups and downs in histogram shape in first row) and some time it causes the existence of new spatial relation such as histogram in the after and before in third row.

For the example figures 5(a) to 5(c), concave object is considered. Figure 5(a) represents object pair, histogram in figure 5(b) is computed by our and use fuzzy operator $O R$. Histogram 


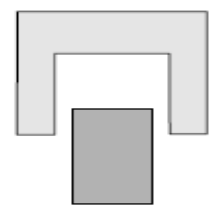

(a)

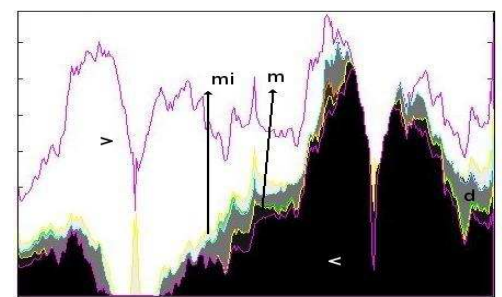

(b)

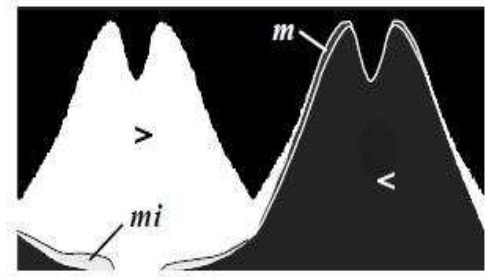

(c)

Figure 5: Comparison of histograms of fuzzy Allen relations with the Matsakis and Nikitenko method and our method for the concave objects. First row represents the object pair and histogram computed by our method and second row represents the histogram computed by Matsakis and Nikitenko method. In our method, sharp change in histogram is due to the change in surface area of objects due to the digitization process (axis are same as in figure 4(b) and 4(c)).

represented in figure 5(c) is computed the method developed by Matsakis and Nikitenko. In certain direction object $A$ is before as well as after, in the meanwhile there exist relation meet and meet_by due to closeness of two segments. This example shows that two segments of an object have the opposite fuzzy Allen relations (a case considered in figure 7(b))

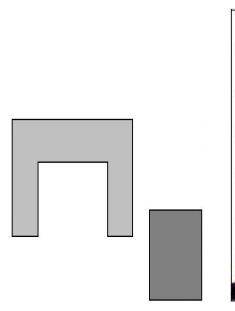

(a)

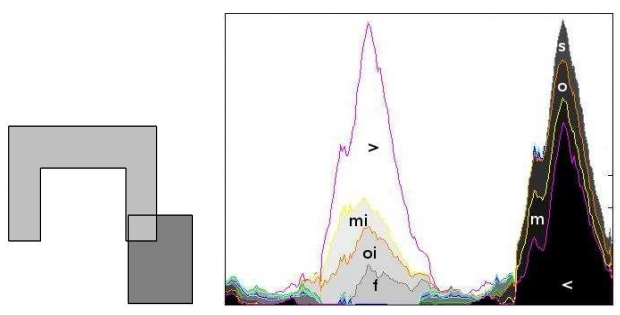

(e)

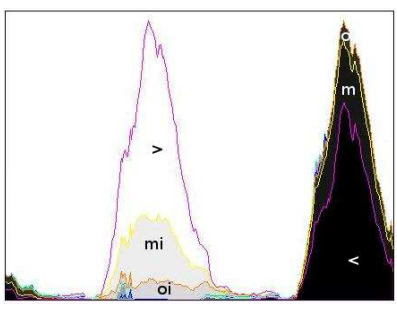

(d)

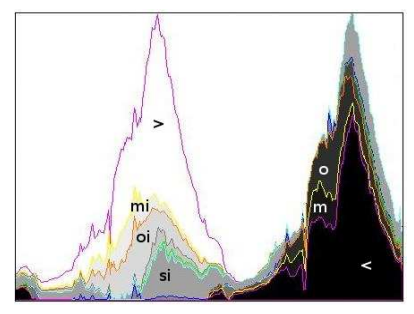

(g)

Figure 6: Concave \& convex object pairs and their histogram of fuzzy Allen relations (axis are same as in figure 4(b) and 4(c)).

In this set of examples (from figure 6(a) to figure 6(g)), argument object is a concave object. As it changes its position, it's topological and directional relation changes with respect to one segment. While the other segment doesn't change it's topological or directional relation information. As a result both segments have opposite Allen relation with the reference segment and resulting histogram represents both relations at the same time. 
Examples given above show that histograms of fuzzy Allen relations are approximately the same while they are computed either with the help of Matsakis and Nikitenko [2] method or polygonal object approximation. Our approach has a certain amount of decrease in the computation time. Computation time with our approach drops from $O(n M \sqrt{M})$ to $O(n N \log (N))$, where $N$ represents the total number of vertices of polygons. For the treatment of longitudinal section, when there exist more than one segment, fuzzy Allen relations are computed between every pair of segments of reference and argument objects, then at the next stage fuzzy OR operator is used to integrate the available fuzzy Allen relations.

\section{Time Complexity}

Efficiency of an algorithm can be measured in terms of execution time. Method for finding the combined topological and directional relations information is to couple the force histograms with the fuzzy Allen relations. Time complexity for computing the combined topological and directional relations information depends upon the following three factors.

1. Algorithm for treatment of longitudinal section

2. Object approximation

3. Equation used for computation of histograms (equation 6 and 7)

These different aspects of time complexity are discussed separately here below. We compare the time complexity of both algorithms and at the end we note the there is a sufficient decrease in the execution time of the modified algorithm.

Time complexity of Algorithm for treatment of longitudinal section: Method for computing the combined topological and directional relations information directly depends upon the force histograms. In this method force histograms are coupled with the fuzzy Allen relations, where algorithm developed by Matsakis and Nikitenko [2] (algorithm1)for fuzzification of segments of a longitudinal section. This algorithm imposes restrictions on the assumption of an object. Its time complexity is added to the time complexity of the force histograms. For better elaboration, the algorithm used by Matsakis and Nikitenko [2] is given below.

Algorithm 1 has the time complexity of order $O\left(n^{3}\right)$. Where $n$ represents the number of segments for a longitudinal section of a line. With the proposed method, this algorithm is replaced by the fuzzy logic connectors, these logic connectors have less time complexity as compared to this algorithm, these fuzzy logic connectors are only used for dealing with the longitudinal section.

Time complexity due to object approximation: In the method of computing the combined topological and directional relations information, force histograms are coupled with the fuzzy Allen relations. Time complexity of algorithm is directly related to the time complexity of force histograms. Time complexity of force histograms for polygonal object approximation is $O(n N \log (N)$ and for raster data it is $O(n M \sqrt{M})$ where $N$ denotes the number of vertices of two polygons and $n$ for number of directions to be computed, $M$ denotes the number of pixels of the processed image (see [26]).

Time complexity of equation 6 and 7 Matsakis and Nikitenko used equation 6 for computing the combined topological and directional relation information. This equation has time 


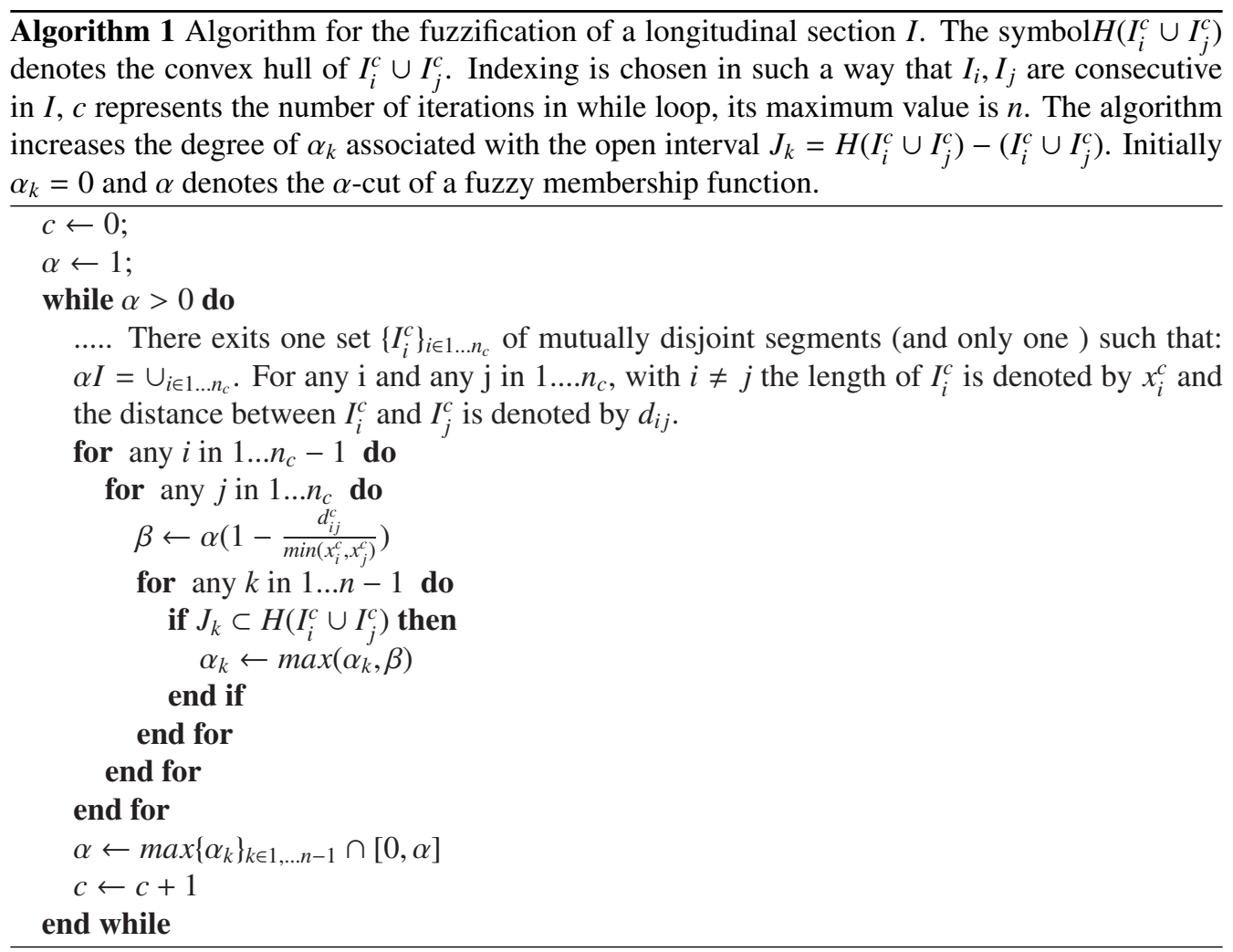

complexity $O\left(n^{3}\right)$ where $n$ represents the total number of segments exist in a longitudinal section. $(m+n$ represents the total number of segments for a longitudinal section and $c$ represents the number of loops in algorithm 1, it maximum value equals to number of disjoint segments). Proposed equation 7 has time complexity $k$ where $k$ represents the total number of vertices of polygons. This shows that the equation 6 has higher time complexity as compared to the time complexity of equation 7 .

Algorithm for fuzzification of longitudinal section used by Matsakis and Nikitenko (Algorithm 1) put limits for the object approximation. Due to this algorithm, time complexity of method for combined topological and directional relations information is $O(n M \sqrt{M})$, where $n$ represents the number of directions $M$ number of pixels to be processed, in this paper we replace the algorithm for fuzzification of segments of a longitudinal section (algorithm 1) with the fuzzy logic connectors. This substitution of algorithm for fuzzification of longitudinal section made it possible to consider objects by its polygon approximation, hence for the method we proposed, time complexity for computing the combined topological and directional relations information drops from $O(n M \sqrt{M})$ to $O(n N \log (N))$, where $N$ represents the total number of vertices of polygons. Obviously $N<<M$ and $n$ is number of directions to be computed, it ranges from 32 to 360 directions. 


\section{Conclusion}

Fuzzy Allen relations can be used to detect and analyze object position in space and these relations have a rich support for defining the fuzzy topological relations. These relations represent fuzziness in relation's semantics, they can also answer the questions that where in space a certain topological relation exists.

Polygonal approximation of objects and application of fuzzy logic connectors simplifies the algorithm given by Matsakis and Nikitenko[2]. This approach decreases its time complexity, it drops down from $O(n M \sqrt{M})$ to $O(n N \log (N)$, where $n$ represents number of directions, $N$ number of vertices of polygons and $M$ number of pixels to be processed, due to using fuzzy logic connectors in lieu of the fuzzification of segments of a longitudinal section developed by Matsakis.

This approach of using fuzzy operator will open new fields of applications for fuzzy logic connectors. This technique can further be developed for defining the dynamic spatial relations in a quantitative way. In this paper, computations for all the directions are calculated for experimental purpose and verification of affine properties will be helpful for affine invariant description of relative object positions in scene and image understanding applications by combined topological and directional relations information. The aim of this paper is to validate formally the method we propose, and for the future work we project to apply this method for real data obtained by video sequences of real applications after extracting objects en their polygonal approximation with help of all the image processing techniques.

\section{References}

[1] J. M. EH. Zahzah, L. Mascarilla, Indexation et Recherche d' Image Fondes Sur Les Relations Spatiales Entre Objets, Traitement du Signal 19(4) (2002) 235-250.

[2] P.Matsakis, D. Nikitenko, Combined Extraction of Directional and Topological Relationship Information from 2D Concave Objects, in Fuzzy Modeling with Spatial Information for Geographic Problems, Springer-Verlag Publications, pp. 15-40, New York.

[3] N. Salamat, E. hadi Zahzah, Spatial relations analysis by using fuzzy operators., in: in proceedings of The International Conference on Computational Science (ICCS(2)), volume 5545 of Lecture Notes in Computer Science, Springer, 2009, pp. 395-404.

[4] C. Millet, I. Bloch, P. Héde, P.-A. Moëllic, Using relative spatial relationships to improve individual region recognition, in: In Proceedings of 2nd European Workshop on the Integration of Knowledge, Semantic and Digital Media Technologies, pp. 119-126.

[5] A. R. Isabelle Bloch, Directional Relative Position Between Objects in Image Processing: A Comparison Between Fuzzy Approaches, pattern Recognition 36 (2003) 1563-1582.

[6] M. J. Egenhofer, K. K. Al-Taha, Reasoning about Gradual Changes of Topological Relationships, in: Proceedings of the International Conference On GIS - From Space to Territory, Springer-Verlag, London, UK, 1992, pp. 196219.

[7] M. J. Egenhofer, J. Sharma, D. M. Mark, A Critical Comparison of The 4-Intersection and 9-Intersection Models for Spatial Relations: Formal Analysis, Auto-Carto 11 (1993) 1-12.

[8] R. K. Goyal, M. J. Egenhofer, Similarity of cardinal directions, in: SSTD, pp. 36-58.

[9] J. Herring, D. M. Mark, M. J. Egenhofer, The 9-intersection: Formalism and its use for natural-language spatial predicates, 1994.

[10] D. Randell, Z. Cui, A. Cohn, A spatial logic based on regions and connection, in: Proc. 3rd Int. Conf. on Knowledge Representation and Reasoning, Morgan Kaufmann, San Mateo, 1992, pp. 165-176.

[11] D. A. Randell, Z. Cui, A. G. Cohn, A spatial logic based on regions and connection, in: KR, pp. 165-176.

[12] K. Liu, W. Shi, Quantitative fuzzy topological relations of spatial objects by induced fuzzy topology, International Journal of Applied Earth Observation and Geoinformation 11 (2009) 38 - 45.

[13] G. K. Palshikar, Fuzzy region connection calculus in finite discrete space domains, Applied Soft Computing 4 (2004) $13-23$.

[14] W. Shi, K. Liu, A fuzzy topology for computing the interior, boundary, and exterior of spatial objects quantitatively in gis, Comput. Geosci. 33 (2007) 898-915. 
[15] X. Tang, Modeling Fuzzy Spatial objects in Fuzzy Topological Spaces with Application to Land Cover Changes, Ph.D. thesis, ITC publications, The Netherlands, 2004.

[16] I. Bloch, Fuzzy relative position between objects in image processing: A morphological approach, IEEE Trans. Pattern Anal. Mach. Intell. 21 (1999) 657-664.

[17] S. Schockaert, M. De Cock, E. Kerre, Modelling nearness and cardinal directions between fuzzy regions, in: Fuzzy Systems, 2008. FUZZ-IEEE 2008. (IEEE World Congress on Computational Intelligence). IEEE International Conference on, pp. $1548-1555$.

[18] K. Miyajima, A. Ralescu, Spatial Organization in 2D Segmented Images: Representation and Recognition of Primitive Spatial Relations, Fuzzy Sets Syst. 65 (1994) 225-236.

[19] Y. Wang, F. Makedon, R-histogram: quantitative representation of spatial relations for similarity-based image retrieval, in: MULTIMEDIA '03: Proceedings of the eleventh ACM international conference on Multimedia, pp. 323-326.

[20] P. Matsakis, L. Wendling, A New Way to Represent the Relative Position between Areal Objects, IEEE Transactions on Pattern Analysis and Machine Intelligence 21 (1999) 634-643.

[21] M. J. Egenhofer, R. D. Franzosa, Point Set Topological Relations , International Journal of Geographical Information Systems 5(2) (1991) 161-174.

[22] A. J.F., Maintaining Knowledge about Temporal Intervals , Communications of the ACM 26(11) (1983) 832-843.

[23] S. Schockaert, M. D. Cock, E. E. Kerre, Imprecise temporal interval relations, in: WILF, pp. 108-113.

[24] M. Grabisch, S. A. Orlovski, R. R. Yager, Fuzzy aggregation of numerical preferences (1998) 31-68.

[25] G. Beliakov, J. Warren, Appropriate choice of aggregation operators in fuzzy decision support systems, 2001.

[26] P. Matsakis, Understanding the spatial organization of image regions by means of force histograms: a guided tour (2002) 99-122.

\section{Annex-A}

Now let's consider that the following situations arise for the segments of a longitudinal section when the objects are concave or objects have disconnected boundary.

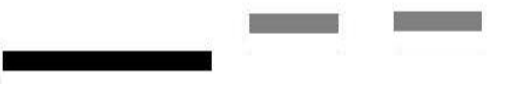

(a)

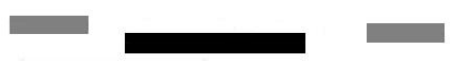

(b)

Figure 7: Different positions of segments in case of longitudinal sections (Argument object in grey and black object represents reference object)

Figure 7(a) We discuss here the cases arising in figure 7(a) with all aggregation operators. For the above cited examples, we use terms $f\left(x_{1}, y_{1}, z\right)$ and $f\left(x_{2}, y_{2}, z\right)$ to express histograms of fuzzy Allen relations for first and second segments, here $x_{1}$ is the length of first segment of the argument object and $x_{2}$ is the length of second segment, similarly $y_{1}$ is the difference between the first segment of argument object and the reference object and $y_{2}$ is the difference between the second segment of argument object and the reference object, in this case consider both $y_{1}>\frac{a_{1}}{2}$ and $y_{2}>\frac{a_{2}}{2}$ where $a_{1}=\min \left(x_{1}, z\right)$ and $a_{2}=\min \left(x_{2}, z\right)$.

$$
\begin{aligned}
& f\left(x_{1}, y_{1}, z\right)=\left(\begin{array}{lllllllllllll}
1 & 0 & 0 & 0 & 0 & 0 & 0 & 0 & 0 & 0 & 0 & 0 & 0
\end{array}\right)^{t} \\
& f\left(x_{2}, y_{2}, z\right)=\left(\begin{array}{lllllllllllll}
1 & 0 & 0 & 0 & 0 & 0 & 0 & 0 & 0 & 0 & 0 & 0 & 0
\end{array}\right)^{t}
\end{aligned}
$$

It means $f_{>}\left(I_{1}, J\right)=1$ and $f_{>}\left(I_{2}, J\right)=1$ and all the other values of histogram are zero. The possible outcomes with the application of different fuzzy operators are 


$$
\begin{aligned}
F_{O R}\left(\theta, A_{\theta}(v), B_{\theta}(v)\right. & =\max \left(f\left(x_{1}, y_{1}, z\right), f\left(x_{2}, y_{2}, z\right)\right) \\
= & (1,0,0,0,0,0,0,0,0,0,0,0,0)^{t} \\
F_{A N D}\left(\theta, A_{\theta}(v), B_{\theta}(v)\right. & =\min \left(f\left(x_{1}, y_{1}, z\right), f\left(x_{2}, y_{2}, z\right)\right) \\
& =(1,0,0,0,0,0,0,0,0,0,0,0,0)^{t} \\
F_{P R O D}\left(\theta, A_{\theta}(v), B_{\theta}(v)\right. & =f\left(x_{1}, y_{1}, z\right) \times f\left(x_{2}, y_{2}, z\right) \\
& =(1,0,0,0,0,0,0,0,0,0,0,0,0)^{t} \\
F_{S U M}\left(\theta, A_{\theta}(v), B_{\theta}(v)=1\right. & -\left(\left(1-f\left(x_{1}, y_{1}, z\right)\right) \times\left(1-f\left(x_{2}, y_{2}, z\right)\right)\right) \\
= & (1,0,0,0,0,0,0,0,0,0,0,0,0)^{t}
\end{aligned}
$$

In this case, the two segments of argument object have the same Allen relations with the reference segment. Both segments behave like the crisp Allen relation. In such a case, all fuzzy operator provide us similar information.

Figure 7(b) The cases arising in figure 7(b) with all aggregation operators. In this case consider $y_{1}<-b-\frac{3 a_{1}}{2}$ and $y_{2}>\frac{a_{2}}{2}$ where $a_{1}=\min \left(x_{1}, z\right), b_{1}=\max \left(x_{1}, z\right)$ and $a_{2}=\min \left(x_{2}, z\right)$ then $f\left(x_{1}, y_{1}, z\right)$ and $f\left(x_{2}, y_{2}, z\right)$ are used to express the histograms of fuzzy Allen relations for first and second segments.

$$
\begin{aligned}
& f\left(x_{1}, y_{1}, z\right)=\left(\begin{array}{lllllllllllll}
0 & 0 & 0 & 0 & 0 & 0 & 0 & 0 & 0 & 0 & 0 & 0 & 1
\end{array}\right)^{t} \\
& f\left(x_{2}, y_{2}, z\right)=\left(\begin{array}{lllllllllllll}
1 & 0 & 0 & 0 & 0 & 0 & 0 & 0 & 0 & 0 & 0 & 0 & 0
\end{array}\right)^{t}
\end{aligned}
$$

The possible outcomes are

$$
\begin{aligned}
F_{O R}\left(\theta, A_{\theta}(v), B_{\theta}(v)\right. & =\max \left(f\left(x_{1}, y_{1}, z\right), f\left(x_{2}, y_{2}, z\right)\right) \\
& =(1,0,0,0,0,0,0,0,0,0,0,0,1)^{t} \\
& =\min \left(f\left(x_{1}, y_{1}, z\right), f\left(x_{2}, y_{2}, z\right)\right) \\
F_{A N D}\left(\theta, A_{\theta}(v), B_{\theta}(v)\right) & (0,0,0,0,0,0,0,0,0,0,0,0,0)^{t} \\
F_{P R O D}\left(\theta, A_{\theta}(v), B_{\theta}(v)\right) & =f\left(x_{1}, y_{1}, z\right) \times f\left(x_{2}, y_{2}, z\right) \\
& =(0,0,0,0,0,0,0,0,0,0,0,0,0)^{t} \\
F_{S U M}\left(\theta, A_{\theta}(v), B_{\theta}(v)\right)= & 1-\left(\left(1-f\left(x_{1}, y_{1}, z\right)\right) \times\left(1-f\left(x_{2}, y_{2}, z\right)\right)\right) \\
= & (1,0,0,0,0,0,0,0,0,0,0,0,1)^{t}
\end{aligned}
$$


The above cited examples explain that, in a particular situation, $A N D$ and $P R O D$ operators cannot be used for the decision making process, when both segments of one longitudinal section of argument object have the same Allen relation with the segment of reference object, all fuzzy operators have the same results. In real situation different cases may arise and segments may have opposite relation as in figure 7(b), then all the information may be lost. These results show that fuzzy conjunction operators give results counter intuitive (both AND, PROD represents the conjunction operators) and the disjunction operators better fits the human intuition and provides here a better fusion of available fuzzy information. The third type of fuzzy operators such as Fuzzy $\gamma$ operator can also be used to make possible contributions of two fuzzy values, but in this case finding compensation values of $\gamma$ is a problem and for each case we have to adjust $\gamma$. When the objects are concave or the objects contain holes, then objects have several segments, called longitudinal section. In such a case, fuzzy Allen relations are calculated for each segment separately then fuzzy connectors are applied for combining information from both segments. 